Research Article

\title{
Propranolol Suppresses Proliferation and Migration of HUVECs through Regulation of the miR-206/VEGFA Axis
}

\author{
Ting Zhang, Yingying Qian, Chunyu Yuan, Yafen Wu, Hua Qian, Hui Lu, Cui Hu, \\ and Wei Li $(D)$
}

Department of Dermatology, Children's Hospital of Soochow University, Suzhou, Jiangsu Province 215025, China

Correspondence should be addressed to Wei Li; liwei9371@suda.edu.cn

Received 26 April 2021; Accepted 24 September 2021; Published 16 October 2021

Academic Editor: Ramoji Kosuru

Copyright (c) 2021 Ting Zhang et al. This is an open access article distributed under the Creative Commons Attribution License, which permits unrestricted use, distribution, and reproduction in any medium, provided the original work is properly cited.

\begin{abstract}
Propranolol has been used in the first-line therapy of infantile hemangioma (IH) for a number of years; however, the mechanisms through which propranolol regulates IH are not yet fully understood. In the present study, microRNA (miRNA/miR) sequencing analysis was performed to identify differentially expressed miRNAs in human umbilical vascular endothelial cells (HUVECs) treated with propranolol. Cell viability and apoptosis were detected using CCK-8 assay and flow cytometry, respectively. Cell migration was assessed using wound healing, Transwell, and tube formation assays. Methylation-specific PCR was then used to investigate the promoter methylation status. The levels of oxidative stress indicators, including superoxide dismutase, glutathione, and malondialdehyde were also detected. Finally, cell cycle analysis was performed using flow cytometry and western blotting. It was observed that propranolol induced the upregulation of miR-206 in HUVECs, which was caused by demethylation of the miR-206 promoter. Moreover, propranolol significantly inhibited the proliferation of HUVECs by inducing apoptosis, while these phenomena were reversed by miR-206 antagomir. VEGFA was found to be a target gene of miR-206. In addition, propranolol notably inhibited the migration and induced G1 arrest of the HUVECs, whereas these results were eliminated by miR-206 antagomir. Collectively, the findings of the present study demonstrated that propranolol may inhibit the proliferation and migration in HUVECs via modulating the miR-206/VEGFA axis. These findings suggest a novel mechanism through which propranolol suppresses the progression of $\mathrm{IH}$.
\end{abstract}

\section{Introduction}

Infantile hemangioma $(\mathrm{IH})$ is the most frequent vascular tumor occurring in infancy, and it affects $\sim 5-10 \%$ of mature neonates [1]. IH begins with the hyperplasia of endothelial cells, which is followed by a period of extensive proliferation and finally the involution phase [2]. In general, $>20 \%$ of the IHs are associated with severe complications, such as ulcerations, visual impairment, airway obstruction, and congestive heart failure [3]. Therefore, accurate diagnostic tools and effective therapies are urgently required. Since the discovery that propranolol can inhibit the growth of severe hemangiomas in 2008 [4], propranolol has been widely used as a first-line therapy [5]. Propranolol is a $\beta$-adrenergic receptor antagonist, which can reduce heart rate and cardiac contractility; thus, it is widely used in the treatment of cardiac-cerebral vascular diseases [6-8]. However, the poor responsiveness and recurrence in some patients require the identification of novel drugs for future therapy $[9,10]$.

MicroRNAs (miRNAs/miRs) are classified as singlestrand noncoding RNAs expressed widely during physiological or pathological processes [11]. In recent years, the role of miRNAs in cancers has been extensively investigated. They are well known to play a role in cancer cell proliferation [12], migration [13], apoptosis [14], and in other cellular processes. Meanwhile, miRNAs are known to mediate the progression of IH. For instance, miR-33a-5p could inhibit the tumorigenesis of IH via targeting HIF1 $\alpha$ [15]; miR196b-5p could facilitate intercellular interaction in IH [16]. However, the role of miRNAs in IH warrants further investigation. In the present study, the differentially expressed miRNAs in propranolol-treated human umbilical vascular endothelial cells (HUVECs) were analyzed. Furthermore, the association between propranolol and the differentially 
expressed miRNAs in HUVECs was investigated. The findings of the present study provide a more theoretical foundation for the propranolol treatment of IH. It is hoped that these findings may also aid in the development of novel drugs for IH therapy.

\section{Materials and Methods}

2.1. Cell Culture and Transfection. HUVECs were obtained from the American Type Culture Collection (ATCC) and cultured in EGM $^{\mathrm{TM}}$ Endothelial Cell Growth Medium (Lonza Group, Ltd.) in a humidified incubator at $37^{\circ} \mathrm{C}$ with $5 \% \mathrm{CO}_{2}$. The cells were then incubated with $0,20,40,60,80$, or $100 \mu \mathrm{M}$ propranolol (Sigma-Aldrich; Merck KGaA) for $48 \mathrm{~h}$, followed by analyses using various assays. miR-206 agomir, miR-206 antagomir, and negative control (Guangzhou RiboBio Co., Ltd.) were transfected into the HUVECs using Lipofectamine $2000^{\circledR}$ (Thermo Fisher Scientific, Inc.).

2.2. Cell Viability Assay. Cell viability was determined using Cell Counting Kit-8 (CCK-8) assay (Nanjing KeyGen Biotech Co., Ltd.). The cells were plated in a 96-well plate and treated with the reagents for $48 \mathrm{~h}$; CCK- 8 reagent was then added to the cells for a further $2 \mathrm{~h}$ at $37^{\circ} \mathrm{C}$. The absorbance was then read at $450 \mathrm{~nm}$ using a Victor $3^{\mathrm{TM}}$ microplate reader (PerkinElmer, Inc.).

2.3. RNA Sequencing and Analysis of Differentially Expressed miRNAs. The HUVECs were treated with or without propranolol and harvested for RNA extraction using TRIzol ${ }^{\circledR}$ reagent (Invitrogen; Thermo Fisher Scientific, Inc.) following the manufacturer's guidelines. The concentration of RNA was then quantified using a Nanodrop 2000 spectrophotometer (Thermo Fisher Scientific, Inc.). RNA library data were generated from the Illumina Hiseq platform (Illumina, Inc.). DESeq2 was used for the analysis of differentially expressed miRNAs [17]. The selection criteria for the upregulated and downregulated miRNAs were a $P$ value $<0.05$ and fold change $>1.2$, and a $P$ value $<0.05$ and fold change $<0.083$, respectively.

2.4. KEGG and GO Analysis for the Protein Targets of Differentially Expressed miRNAs. The protein targets of differentially expressed miRNAs were analyzed using miRTarBase. ClusterProfiler software was used for Gene Ontology (GO) and Kyoto Encyclopedia of Genes and Genomes (KEGG) analysis. The GO database (http://www.geneontology.org/) was used to annotate the functions of protein targets of differentially expressed miRNAs. The KEGG database (https://www .genome.jp/kegg/) was used to determine the potential pathways of the protein targets of differentially expressed miRNAs.

2.5. Reverse Transcription-Quantitative PCR (RT-qPCR). RNA extraction from HUVECs was performed using TRIpure RNA Extraction Reagent (ELK Biotechnology, Co., Ltd.). First, RNA was reverse transcribed into cDNA using the EntiLink cDNA Synthesis kit (ELK Biotechnology, Co., Ltd.). The mRNA level was then determined using SYBRGreen (ELK Biotechnology, Co., Ltd.) on a StepOne ${ }^{\mathrm{TM}}$ PCR System (Thermo Fisher Scientific, Inc.). The specific primers used were as follows: miR-206 forward, $5^{\prime}$-TGGAATGTAAG GAAGTGTGTGG-3' and reverse, $5^{\prime}$-CTCAACTGGTGTCG TGGAGTC-3'; VEGFA forward, $5^{\prime}$-GAACTTTCTGCTGT CTTGGGTG-3' and reverse, $5^{\prime}$-GGCAGTAGCTGCGCTG ATAG-3' . U19 and $\beta$-actin were used as the internal controls for miR-206 and VEGFA, respectively. The relative mRNA levels were quantified using the $2^{-\Delta \Delta \mathrm{Cq}}$ method [18].

2.6. Methylation-Specific PCR. HUVECs were treated with $80 \mu \mathrm{M}$ propranolol for $48 \mathrm{~h}$ and then harvested for DNA extraction using the Genomic DNA Extraction kit (ELK Biotechnology, Co., Ltd.). Subsequently, DNA was subjected to bisulfite modification following the manufacturer's protocols (Methylation-Gold kit; Zymo Research Corp.). The primers for methylated (M) and unmethylated (U) PCR were synthesized by Sangon Biotech, Co., Ltd. and were as follows: $M$ forward, 5' -TTGTATAAGAATAAGTTAGGGAAACG-3' ${ }^{\prime}$ and reverse, $5^{\prime}$-CCCAAACAAAAAACTCTTAACG-3'; and U forward, $5^{\prime}$-GTTGTATAAGAATAAGTTAGGGAAATG-3' and reverse, 5' -TACCCAAACAAAAAACTCTTAACA-3'. Finally, $2 \%$ agarose gel was used to separate the PCR products.

2.7. Flow Cytometry. HUVECs were collected and resuspended in PBS supplemented with $0.5 \%$ FBS and $2 \mathrm{mM}$ EDTA. After washing with PBS, the cells were stained using the Annexin V-FITC/PI double staining kit (Nanjing KeyGen Biotech. Co. Ltd.). PI/RNase staining buffer (BD Biosciences) was used for cell cycle analysis. All steps were performed according to the manufacturer's instructions. Detection was performed using a flow cytometer (BD Biosciences) within $1 \mathrm{~h}$, and the data were analyzed using FlowJo 7.6 software (FlowJo LLC).

2.8. Wound Healing Assay. HUVECs were seeded in a 12well plate until they reached a confluency of $80 \%$. A scratch was then made in a straight line in the cell layer using a $100 \mu$ l pipette tip. The cells were then washed twice with PBS to remove the suspended cells and cultured in complete medium. The same scratch area was photographed at $0 \mathrm{~h}$ and $48 \mathrm{~h}$ under a microscope (Olympus Corporation). Finally, the wound healing rate was calculated according to the change in the wound area [19].

2.9. Transwell Assay. The Transwell migration assay was performed using 24-well Transwell chambers with an $8 \mu \mathrm{m}$ pore size (Corning, Inc.). First, $200 \mu$ l HUVECs in serumfree medium was added to the upper chamber of the well, and $600 \mu \mathrm{l}$ complete medium was added to the lower chamber. The unmigrated cells on the upper surface were then removed using a cotton swab, and the migrated cells on the lower surface were stained with $0.2 \%$ crystal violet following $24 \mathrm{~h}$ of incubation. The images were captured under a light microscope (Olympus Corporation).

2.10. Tube Formation Assay. Matrigel ${ }^{\mathrm{TM}}$ (BD Biosciences) was applied to assess the tube formation ability of the HUVECs. The 6-well plates were precoated with Matrigel at $37^{\circ} \mathrm{C}$ for $1 \mathrm{~h}$. The HUVECs were then seeded into these wells and incubated for $24 \mathrm{~h}$. The images of tube formation 


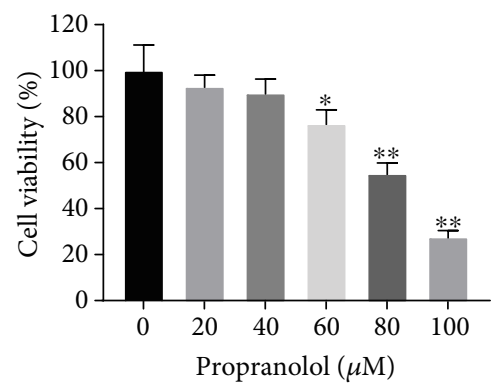

(a)

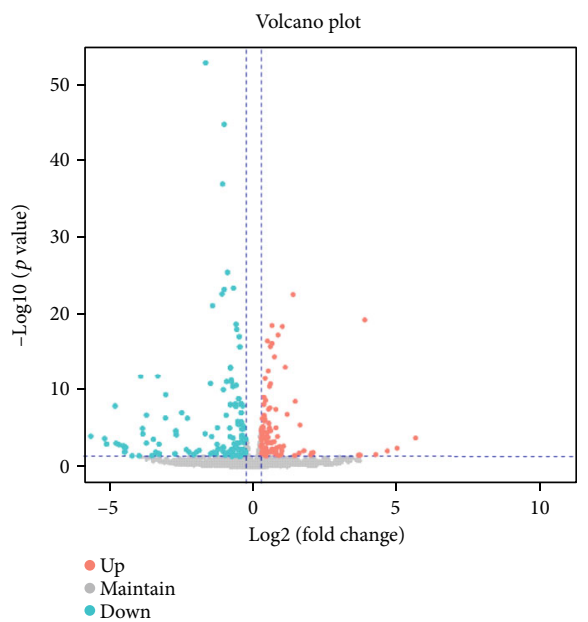

(c)

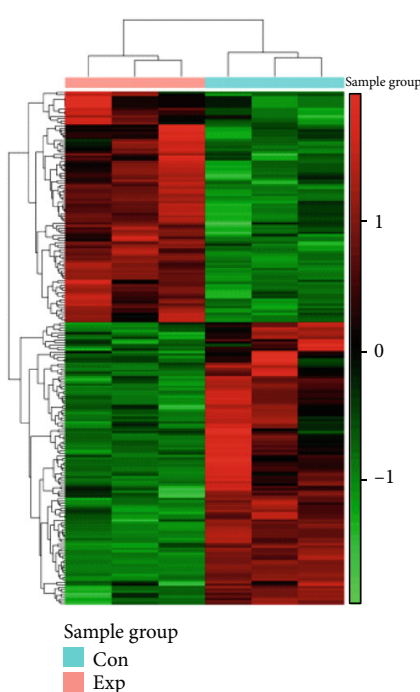

(b)

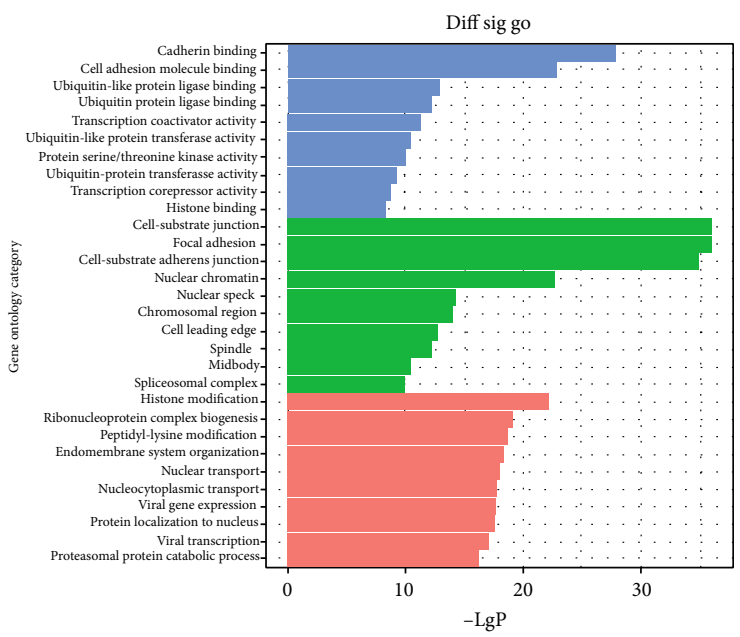

Type

Biological process

Cellular componen

Molecular function

(d)

Figure 1: Continued. 


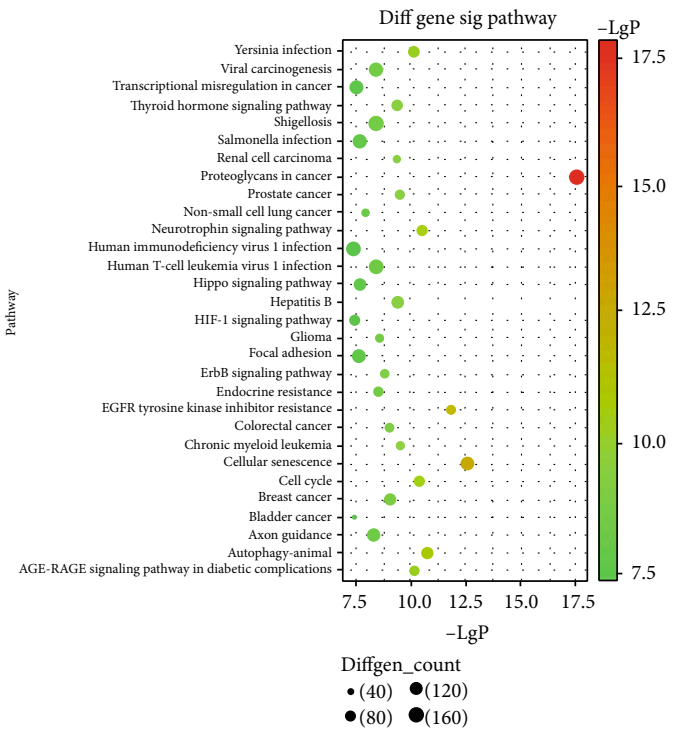

(e)

FIGURE 1: DEG analysis of HUVECs treated with propranolol. (a) Cell viability was determined by CCK- 8 assay. $N=3,{ }^{*} P<0.05,{ }^{* *} P<0.01$ , all comparing with the $0 \mu \mathrm{M}$ propranolol group. (b) Heat map showing the DEGs in cells treated with $80 \mu \mathrm{M}$ propranolol comparing with control cells. Red represents upregulated genes, green represents downregulated genes, and black represents unchanged genes, $N=3$. (c) Volcano plot map showing the significantly upregulated $(P$ value $<0.05$, fold change $>1.2)$ and downregulated $(P$ value $<0.05$, fold change $<0.833$ ) miRNAs. (d) GO enrichment analysis of protein targets of DEGs. (e) KEGG pathway analysis of protein targets of DEGs.

were captured under a microscope (Olympus Corporation). Branching points and capillary lengths from three random fields were analyzed using WimTube software (Wimasis).

2.11. Dual-Luciferase Reporter Assay. The potential association between miR-206 and VEGFA was predicted using bioinformatics tools, miRDB (http://www.mirdb.org) and TargetScan (http://www.targetscan.org). The VEGFA 3'UTR sequences containing the wild-type or mutant miR206 binding sites were then constructed and inserted into the pGL6 luciferase reporter vector (Beyotime Institute of Biotechnology). HUVECs were cotransfected with the luciferase reporter vector plus miR-206 agomir or control using Lipofectamine $2000^{\circledR}$ (Thermo Fisher Scientific, Inc.). Following $48 \mathrm{~h}$ of transfection, the cells were harvested for luciferase activity detection following the instructions provided with the Dual-Luciferase Reporter Assay System (Promega Corporation).

2.12. Western Blot Analysis. Total protein from was extracted from the HUVECs and electrophoresed with 12\% SDS-PAGE, followed by transfer onto PVDF membranes (Invitrogen; Thermo Fisher Scientific, Inc.). Subsequently, the membranes were blocked with $5 \%$ skim milk and then incubated with the following primary antibodies at $4^{\circ} \mathrm{C}$ overnight: VEGFA, pAKT, p-ERK (1:500), AKT, ERK, CDK4, Cyclin D1, and cleaved caspase-3 $(1: 1,000)$. After washing with TBST three times, the membranes were incubated with specific secondary antibodies labeled with HRP for $1 \mathrm{~h}$. Finally, the membranes were visualized using the ECL chemiluminescent substrate kit (Thermo Fisher Scientific, Inc.) and analyzed using ImageJ software (National Institutes of Health). The antibodies were all obtained from Cell Signaling Technology, Inc. $\beta$-Actin was used as the internal standard.

2.13. Detection of Oxidative Stress Indicators. The indicators of oxidative stress, including superoxide dismutase (SOD) activity, glutathione (GSH), and malondialdehyde (MDA) content, were examined. All assays were performed as per the manufacturer's protocols (Nanjing Jiancheng Bioengineering Institute).

2.14. Statistical Analysis. All experiments were repeated at least three times. The data were analyzed using GraphPad Prism software (GraphPad Software Inc.) and presented as the mean \pm SD. The Student's $t$-test (two-tailed) and oneway ANOVA were applied to compare results between 2 groups or among multiple groups. $P<0.05$ was considered to indicate a statistically significant difference.

\section{Results}

3.1. Profiling of Differentially Expressed miRNAs in PropranololTreated HUVECs. HUVECs were treated with serial concentrations of propranolol $(0,20,40,60,80$, or $100 \mu \mathrm{M})$. The results of the CCK- 8 assay revealed that propranolol treatment led to a concentration-dependent decrease in cell viability at $48 \mathrm{~h}$ (Figure 1(a)). Subsequently, miRNA-seq was performed on three independent groups of HUVECs treated with or without $80 \mu \mathrm{M}$ propranolol. As shown in the clustering analysis of the differentially expressed miRNAs (Figure 1(b)), 97 miRNAs were identified to be upregulated $(P$ value $<0.05$; fold change $>1.2$ ), and 119 miRNAs were downregulated $(P$ value $<0.05$; fold change $<0.833$ ). Additionally, these differentially 


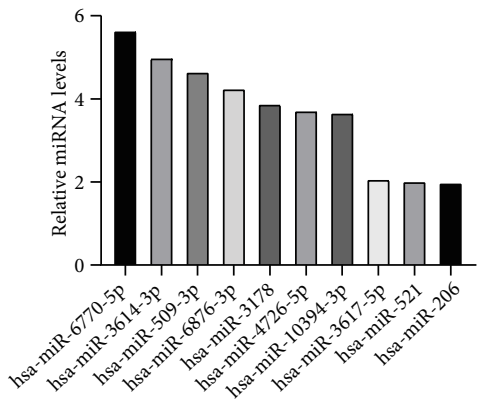

(a)

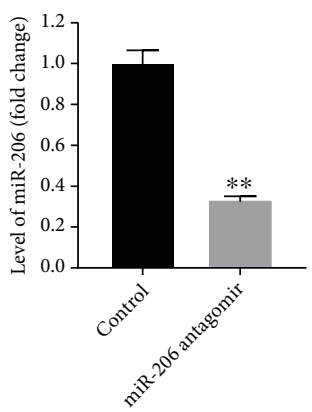

(d)

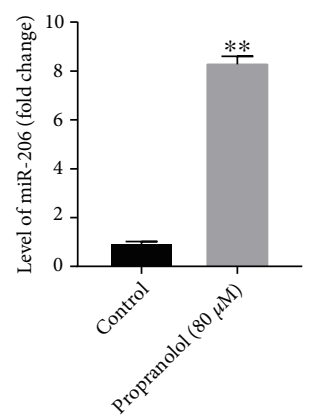

(b)

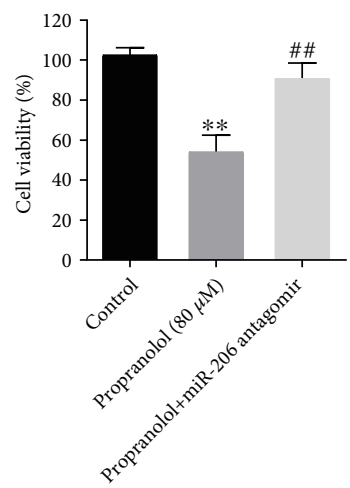

(e)

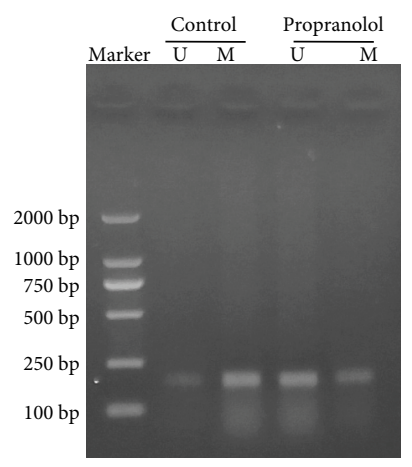

(c)
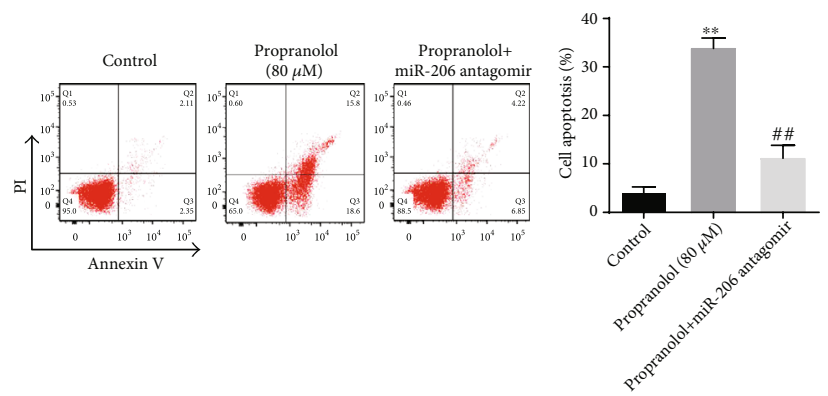

(f)

FIgURe 2: Propranolol inhibits survival of HUVECs through demethylation of pre-miR-206. (a) Top-ten upregulated miRNAs in propranolol-treated $(80 \mu \mathrm{M})$ HUVECs comparing with control cells. (b) HUVECs were treated with $80 \mu \mathrm{M}$ propranolol for $48 \mathrm{~h}$, and the mRNA level of miR-206 was determined by RT-qPCR. (c) Methylation and unmethylation status of the pre-miR-206 gene were measured by MS-PCR. (d) The mRNA level of miR-206 in cells was determined by RT-qPCR after miR-206 antagomir transfection. (e) Cell viability was detected by CCK-8 kit. (f) Cell apoptosis was analyzed on flow cytometry after Annexin V/PI double staining. ${ }^{* *} P<$ 0.01 , comparing with the control group; ${ }^{\# \#} P<0.01$, comparing with the propranolol group; $n=3$.

expressed miRNAs were presented in a volcano plot (Figure 1(c)). GO and KEGG analyses were then performed to analyze the enrichment functions and pathways of the upregulated target genes. GO analysis revealed that the upregulated targets were mostly involved in "cadherin binding," "cell substrate junction," and "histone modification" (Figure 1(d)). KEGG analysis revealed that the upregulated targets were mostly focused in "Proteoglycans in cancer," "Shigellosis," "cellular senescence," and "Cell cycle" (Figure 1(e)).

\subsection{Propranolol Exerts Antiproliferative Effects on HUVECs} through Demethylation of the miR-206 Promoter. As shown by the results obtained for the differentially expressed miRNAs, miR-206 was one of the markedly upregulated miRNAs in the propranolol-treated HUVECs (Figure 2(a)). miR-206 has been demonstrated to play a key role in the vascular function of various cancer cells [20,21]; thus, the association between miR-206 and propranolol in HUVECs was then investigated in the following experiments. The results of RT-qPCR further demonstrated that treatment with $80 \mu \mathrm{M}$ propranolol significantly upregulated the miR-
206 level in the cells (Figure 2(b)). As is known, methylation in the CpG island can result in the downregulation of miRNAs [22]. Thus, methylation-specific PCR was performed to verify the methylation status of the miRNA-206 promoter. Compared with the control cells, propranolol treatment led to a decrease in the $\mathrm{M}$ promoter and an increase in the $\mathrm{U}$ promoter (Figure 2(c)). In order to further determine the role of miR-206 in propranolol-treated HUVECs, miR-206 antagomir was transfected into the cells. RT-qPCR confirmed that miR-206 antagomir notably decreased the expression of miR-206 in HUVECs (Figure 2(d)). Furthermore, propranolol significantly inhibited the growth of HUVECs by inducing apoptosis (Figures 2(e) and 2(f)), while this phenomenon was completely revered by miRNA-206 antagomir. Taken together, propranolol significantly inhibited the growth of HUVECs via demethylation of the miR-206 promoter.

3.3. Downregulation of miR-206 Reverses the PropranololInduced Inhibition of the Migration of HUVECs. A previous study suggested that propranolol can inhibit the migration of HUVECs [23]. In order to further explore the role of 

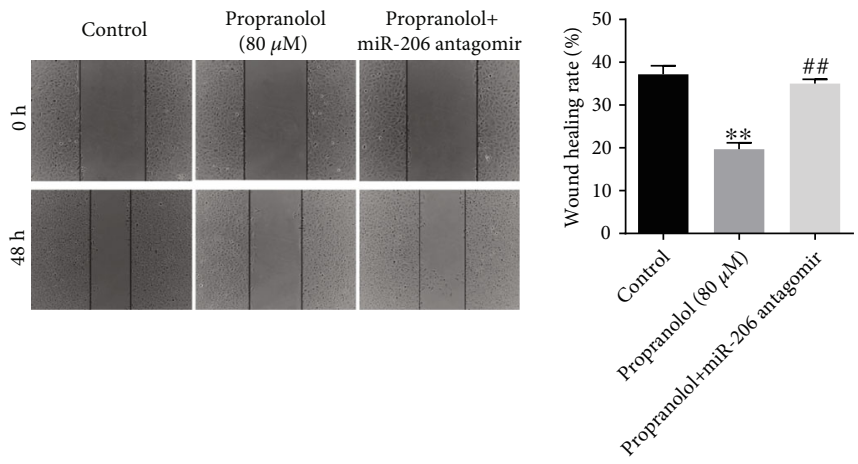

(a)

(b)
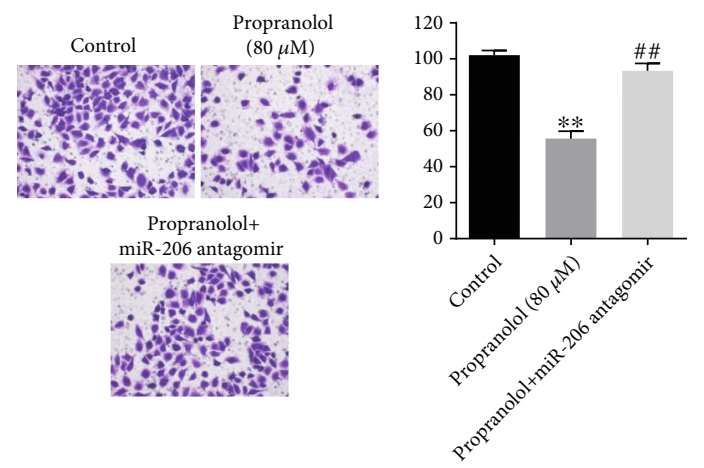

(c)
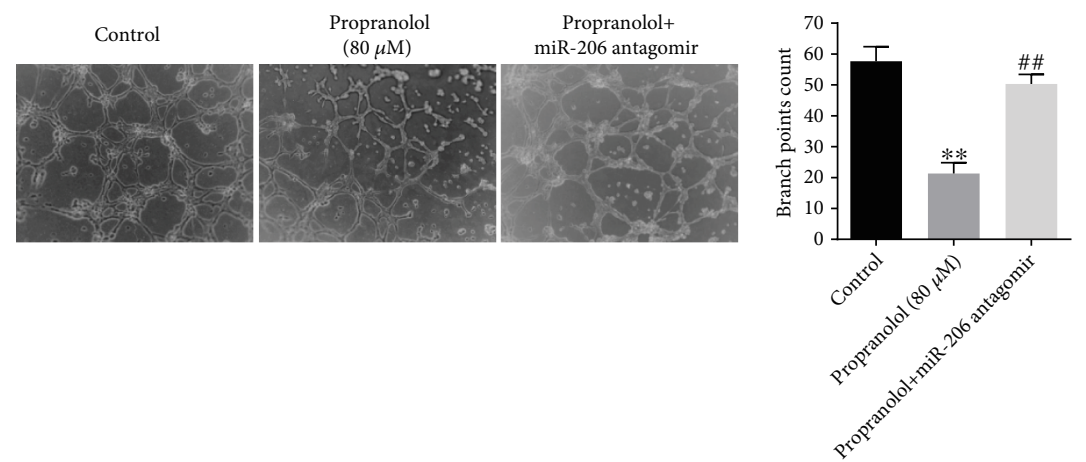

(e)

(f)

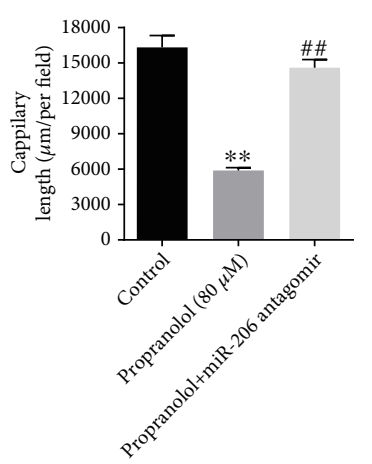

(g)

FIGURE 3: Downregulation of miR-206 reverses propranolol-induced inhibition of cell migration in HUVECs. HUVECs were divided into three groups: control, $80 \mu \mathrm{M}$ propranolol, $80 \mu \mathrm{M}$ propranolol + miR-206 antagomir. (a) and (b) Representative scratching area of cells and the calculated wound healing rate were indicated. (c) and (d) Migrated cells were stained with $0.2 \%$ crystal violet and counted at 3 random fields. (e) Representative images of tube formation were captured by microscopy at $24 \mathrm{~h}$. (f) and (g) Branch points counts and capillary length were calculated at 3 random fields. ${ }^{* *} P<0.01$, comparing with the control group; ${ }^{\# \#} P<0.01$, comparing with the propranolol group; $n=3$. 


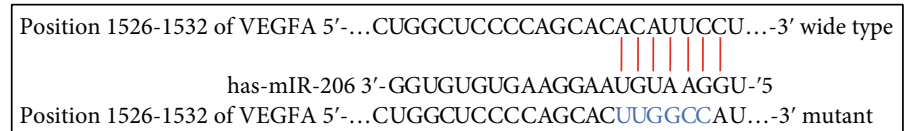

(a)

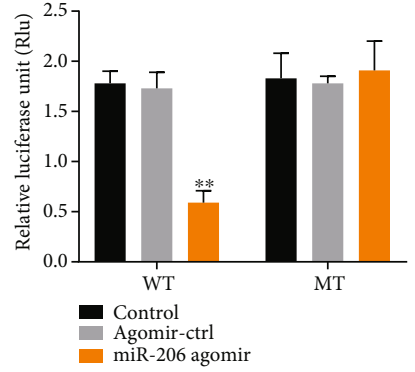

(b)

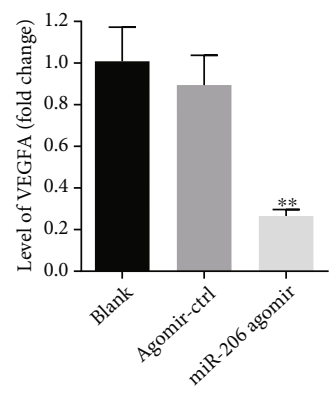

(c)

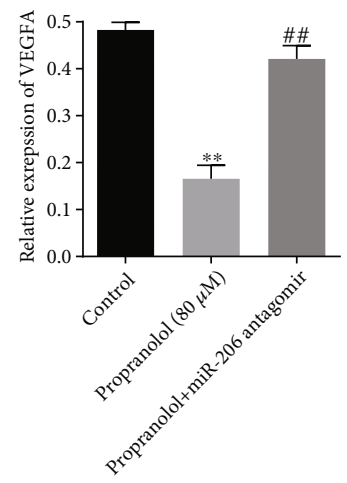

(e)

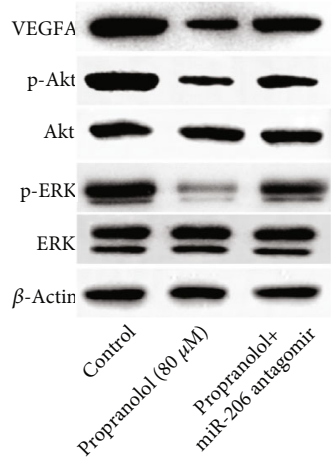

(d)

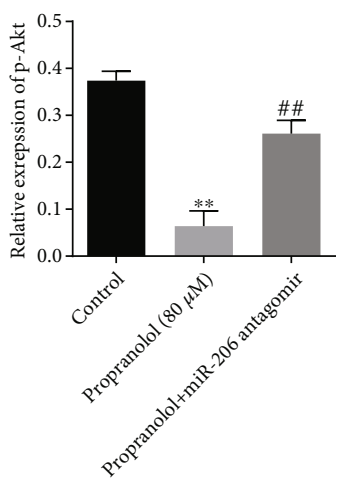

(f)

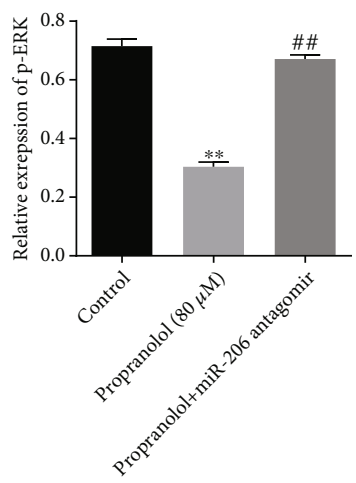

(g)

Figure 4: VEGFA is a target gene of miR-206. (a) Predicted binding sequences between miR-206 and VEGFA. (b) The relationship between miR-206 and VEGFA was verified by dual-luciferase reporter assay. ${ }^{* *} P<0.01$, comparing with the agomir control group; ${ }^{\# \#} P<0.01$, comparing with the propranolol group; $n=3$. (c) The mRNA level of VEGFA in HUVECs was detected with RT-qPCR after miR-206 antagomir transfection. (d)-(g) The protein levels of VEGFA, p-Akt, Akt, p-ERK, and ERK were determined by western blotting, and the relative expression of proteins was normalized with $\beta$-actin. ${ }^{* *} P<0.01$, comparing with the control group; ${ }^{\# \#} P<0.01$, comparing with the propranolol group; $n=3$. 


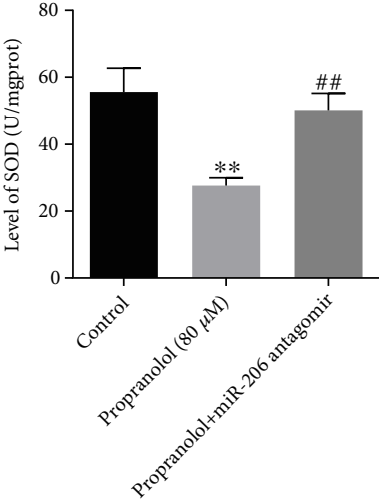

(a)

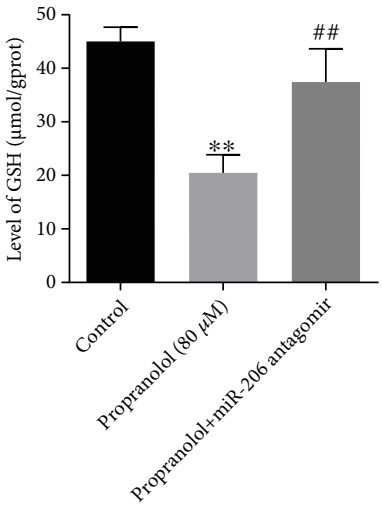

(b)

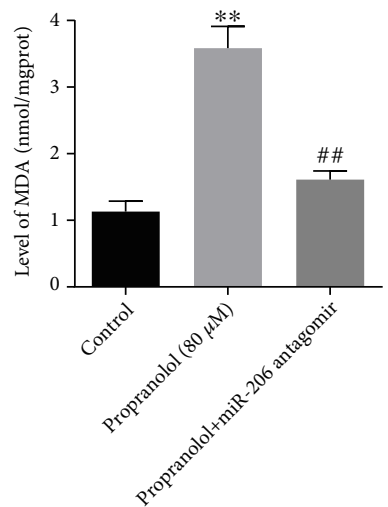

(c)

FIGURE 5: Downregulation of miR-206 abolishes the antioxidant capacity of propranolol in HUVECs. Cells were treated with propranolol or propranolol+miR-206 antagomir, the changes of (a) SOD activity, (b) GSH level, and (c) MDA level in HUVECs were measured, respectively. ${ }^{* *} P<0.01$, comparing with the control group; ${ }^{\# \#} P<0.01$, comparing with the propranolol group; $n=3$.

miR-206 in the propranolol-mediated inhibition of cell migration, Transwell, wound healing, and tube formation assays were conducted. As was expected, propranolol significantly inhibited the wound healing rate of HUVECs, while miR-206 antagomir notably reversed this effect (Figures 3(a) and 3(b)). Likewise, Transwell assay further confirmed that miR-206 antagomir reversed the propranolol-induced inhibition of cell migration (Figures 3(c) and 3(d)). In addition, the results of tube formation assay indicated that propranolol significantly decreased the number of branch points and the length of capillaries, whereas these phenomena were notably reversed by miR-206 antagomir. Taken together, the propranololinduced inhibition of cell migration was reversed by miR-206 inhibition.

3.4. VEGFA Is a Potential Binding Target of miR-206. To further investigate the role of miR-206 in propranolol-treated HUVECs, the potential target of miR-206 was searched using the miRDB and TargetScan databases. Both databases revealed that VEGFA may be a possible target gene of miR-206. The binding sequences between miR-206 and the $3^{\prime}$-UTR of VEGFA are presented in Figure 4(a). Furthermore, dualluciferase reporter assay revealed that miR-206 agomir significantly reduced the luciferase activity of wild-type VEGFA, while it had no effect on the mutant one (Figure 4(b)). More- over, miR-206 agomir notably suppressed the level of VEGFA in HUVECs (Figure 4(c)). Additionally, propranolol decreased the levels of VEGFA, p-AKT, and p-ERK in HUVECs, whereas these phenomena were significantly reversed by miR-206 antagomir (Figures 4(d)-4(g)). All these results illustrated propranolol suppressed the proliferation and migration of HUVECs through the regulation of the miR-206/VEGFA axis.

3.5. Downregulation of $m i R-206$ Abolishes the Antioxidant Capacity of Propranolol in HUVECs. Since propranolol has been demonstrated to prevent angiogenesis via abolishing oxidative stress [24], indicators of oxidative stress were then detected. As shown in Figures 5(a) and 5(b), the activity of SOD and the contents of GSH were decreased following propranolol treatment; however, miR-206 antagomir reversed these effects. In addition, the level of MDA was notably increased by propranolol, and this effect was notably reversed by miR-206 inhibition. All these data suggested that propranolol exerted its antioxidant effects on HUVECs through the modulation of miR-206.

3.6. Downregulation of miR-206 Eliminates PropranololInduced Cell Cycle Arrest of HUVECs. As revealed by KEGG pathway analysis, the targets of the differentially expressed miRNAs exhibited a close association with "Cell cycle," 

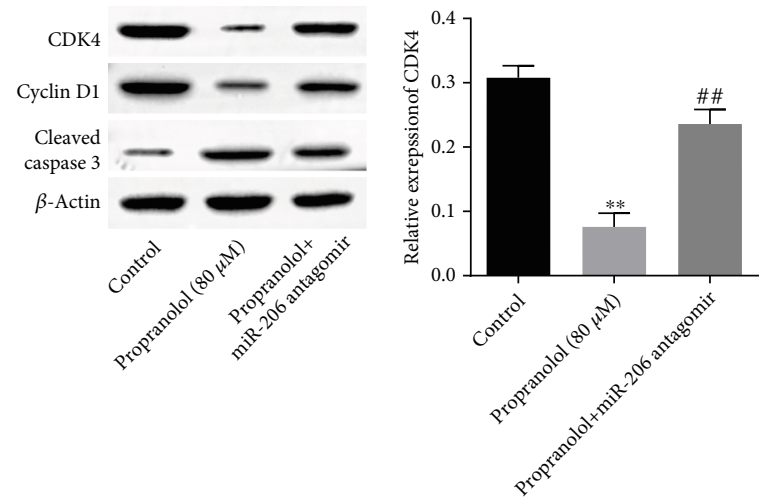

(a)

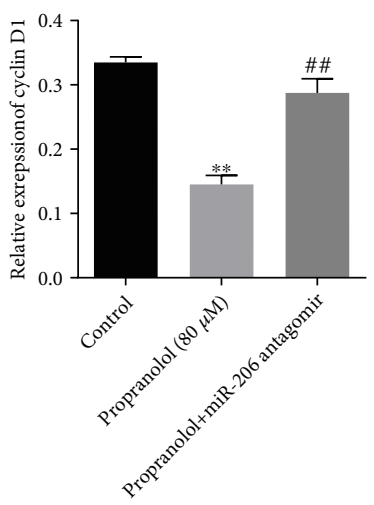

(c)
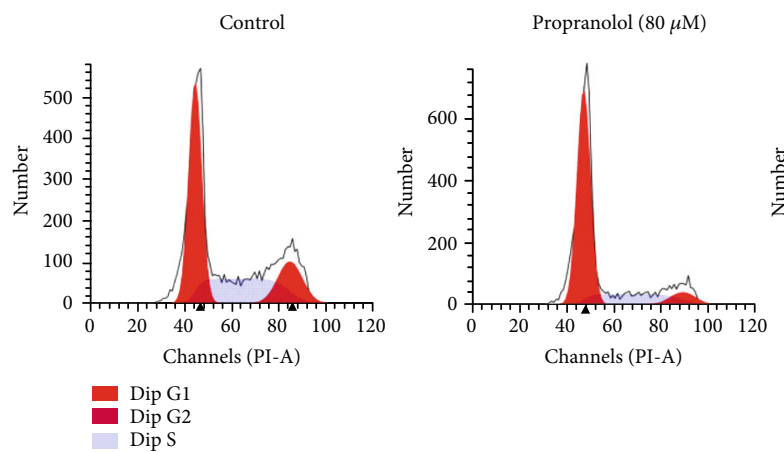

(b)

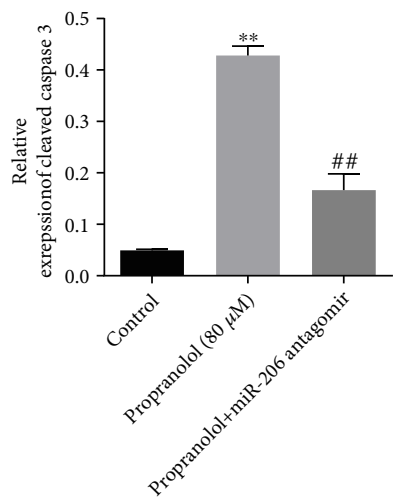

(d)

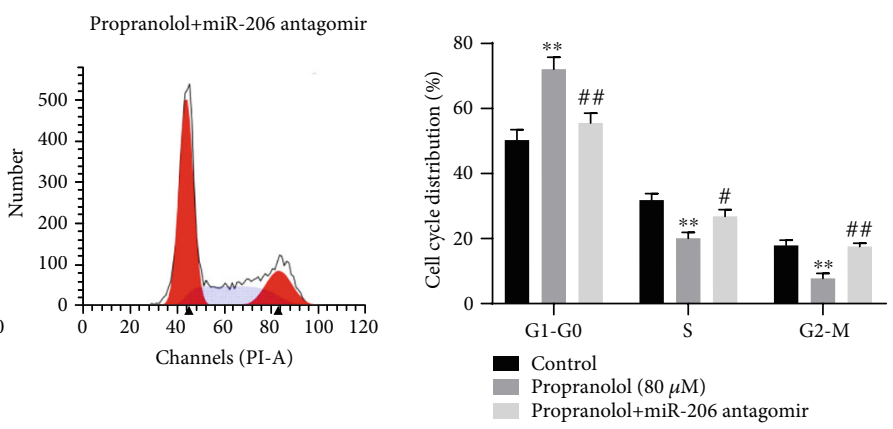

(e)

FIGURE 6: Downregulation of miR-206 eliminates propranolol-induced cell cycle arrest in HUVECs. Cells were treated with propranolol or propranolol+miR-206 antagomir. (a) The protein levels of CDK4, Cyclin D1, and cleaved caspase 3 were detected by western blotting. (b)-(d) The relative expression levels of CDK4, Cyclin D1, and cleaved caspase 3 were quantified by normalizing to $\beta$-actin. (e) Cell cycle distribution was analyzed by flow cytometry, and the result was quantified by flowjo software. ${ }^{* *} P<0.01$, comparing with the control group; ${ }^{\# \#} P<0.01$, comparing with the propranolol group; $n=3$.

and previous reports revealed that miR-206 could inhibit the cancer cell growth via mediation of cell cycle proteins [25, 26]. Thus, cell cycle behavior was then detected in the following experiment. As was expected, the expression levels of CDK4 and cyclin D1 were decreased following propranolol treatment, while miR-206 antagomir significantly reverse these effects (Figures 6(a) and 6(b)). On the other hand, the propranolol-induced upregulation of cleaved caspase- 3 expression was notably reversed by miR-206 antagomir (Figure 6(c)). Additionally, cell cycle analysis revealed that G1 arrest induced by propranolol in the HUVECs was notably eliminated by miR-206 inhibition. Taken together, these data suggested that the propranolol-induced cell cycle arrest of HUVECs was eliminated by miR-206 inhibition.

\section{Discussion}

For decades, it has been known that miRNAs function as key regulators of vascular diseases. In 2016, Strub et al. [27], for the first time, found that miRNA-C19MC was a biomarker of IH. The level of circulating C19MC was associated with the IH tumor size and the response to propranolol treatment [27]. Thereafter, other studies found that miRNAs regulated the process of IH through various mechanisms. For example, 
Li et al. [28] found that propranolol treatment led to the downregulation of miR-382 via the PTEN/AKT/mTOR pathway in XPTS-1 cells. Mong et al. also revealed that the LIN28B/Let-7 signaling axis was involved in the propranolol-induced involution of $\mathrm{IH}$ [29]. As is known, due to its ability to decrease DNA synthesis [30], miR-206 has been shown to reduce angiogenesis [31], thus, suggesting that it may effectively inhibit cancer development $[20,32]$. Recent studies have demonstrated that miR-206 can inhibit the proliferation, invasion, and migration of a number of cancer cells [20,33,34]. Similar to these studies, the present study found that miR-206 was involved in the antiangiogenic effects of propranolol on HUVECs.

Tumor angiogenesis involves endothelial cell proliferation and migration, which are activated through the VEGF/VEGFR pathway $[35,36]$. As aforementioned, miR-206 plays an antiangiogenic role in different tumor types. There is evidence to indicate that miR-206 targets the VEGFA/CCL2 signaling pathway to inhibit tumor progression [37]. Another study found miR-206 suppressed the Met/ERK/Elk-1/HIF$1 \alpha$ /VEGF-A pathway in CCL19-mediated colorectal cancer cells [21]. In the present study, it was found that VEGFA was a target of miR-206, and that miR-206 antagomir reversed the propranolol-induced downregulation of VEGFA in HUVECs. Another study also demonstrated that propranolol reduced the expression of VEGF and VEGF-A via the downregulation of miR-4295 in HUVECs [23]. Thus, there may be other signaling pathways participating in this process, which warrants further investigation into this matter in the future.

In the present study, an important finding was that the "Cell cycle" was involved in the upregulated miRNAs, as revealed using KEGG analysis. The role of the cyclin D/CDK axis in angiogenesis has already been documented [38]. Additionally, researchers have found that miRNAs affect the progression of the cell cycle by targeting the CDK1 and 4/6 genes [39] or E2F transcription factor 8 [40]. Although the cell cycle-related proteins were not found to be the direct target of miR-206 in the present study, cell cycle arrest was observed in the propranolol-treated HUVECs.

Another interesting finding of the present study was that propranolol treatment decreased the methylation level of pre-miR-206. DNA hypermethylation in the promoter is the most frequent mechanism leading to downregulated miRNAs [41]. Previously, researchers have found aberrant methylation status of miRNAs in various types of cancer $[42,43]$, and methylated miRNAs have been shown to be involved in cancer cell proliferation $[44,45]$. This finding may lead to the better understanding of the mechanisms through which propranolol regulates the progression of $\mathrm{IH}$.

\section{Conclusions}

In conclusion, the findings of the present study demonstrated that propranolol may suppress the proliferation and migration of HUVECs through the regulation of the miR-206/VEGFA axis. These findings partly explain the mechanisms through which propranolol regulates the progression of $\mathrm{IH}$ and may aid in the development of novel therapies and drugs in the future.

\section{Data Availability}

The datasets used and/or analyzed during the current study are available from the corresponding author on reasonable request.

\section{Conflicts of Interest}

The authors declare that there are no competing interests.

\section{Authors' Contributions}

Ting Zhang, Yingying Qian, and Chunyu Yuan performed most of the experiment. Yafen Wu, Hua Qian, Hui Lu, and Cui $\mathrm{Hu}$ performed part of the experiments and analyzed the data. Ting Zhang wrote the manuscript. Wei Li supervised the study and revised the manuscript.

\section{Acknowledgments}

This study was supported by the National Natural Science Foundation of China (no. 81301380) and Basic research on the application of people's livelihood technology in medical and health care in Suzhou (no. sys2018076).

\section{References}

[1] Z. B. Zhou, G. X. Huang, Q. Fu et al., "circRNA.33186 contributes to the pathogenesis of osteoarthritis by sponging miR127-5p," Molecular Therapy, vol. 27, no. 3, pp. 531-541, 2019.

[2] C. Léauté-Labrèze, J. I. Harper, and P. H. Hoeger, "Infantile haemangioma," The Lancet, vol. 390, no. 10089, pp. 85-94, 2017.

[3] A. N. Haggstrom, B. A. Drolet, E. Baselga et al., "Prospective study of infantile hemangiomas: clinical characteristics predicting complications and treatment," Pediatrics, vol. 118, no. 3, pp. 882-887, 2006.

[4] C. Léauté-Labrèze, E. Dumas de la Roque, T. Hubiche, F. Boralevi, J. B. Thambo, and A. Taïeb, "Propranolol for severe hemangiomas of infancy," The New England Journal of Medicine, vol. 358, no. 24, pp. 2649-2651, 2008.

[5] A. L. Marqueling, V. Oza, I. J. Frieden, and K. B. Puttgen, "Propranolol and infantile hemangiomas four years later: a systematic review," Pediatric Dermatology, vol. 30, no. 2, pp. 182-191, 2013.

[6] H. J. Duff, L. B. Mitchell, and D. G. Wyse, "Antiarrhythmic efficacy of propranolol: comparison of low and high serum concentrations," Journal of the American College of Cardiology, vol. 8, no. 4, pp. 959-965, 1986.

[7] G. G. Geyskes, P. Boer, J. Vos, F. H. Leenen, and E. J. Mees, "Effect of salt depletion and propranolol on blood pressure and plasma renin activity in various forms of hypertension," Circulation Research, vol. 36, no. 6, pp. 248-256, 1975.

[8] D. Lee, E. Boscolo, J. T. Durham, J. B. Mulliken, I. M. Herman, and J. Bischoff, "Propranolol targets the contractility of infantile haemangioma-derived pericytes," The British Journal of Dermatology, vol. 171, no. 5, pp. 1129-1137, 2014.

[9] L. Chang, D. Lv, Z. Yu et al., "Infantile hemangioma: factors causing recurrence after propranolol treatment," Pediatric Research, vol. 83, no. 1, pp. 175-182, 2018. 
[10] J. Goswamy, M. P. Rothera, and I. A. Bruce, "Failure of propranolol in the treatment of childhood haemangiomas of the head and neck," The Journal of Laryngology and Otology, vol. 125, no. 11, pp. 1164-1172, 2011.

[11] S. M. Hammond, "An overview of microRNAs," Advanced Drug Delivery Reviews, vol. 87, pp. 3-14, 2015.

[12] R. M. Chang, S. Xiao, X. Lei, H. Yang, F. Fang, and L. Y. Yang, "miRNA-487a promotes proliferation and metastasis in hepatocellular carcinoma," Clinical Cancer Research, vol. 23, no. 10, pp. 2593-2604, 2017.

[13] J. Zhu, B. Liu, Z. Wang et al., "Exosomes from nicotinestimulated macrophages accelerate atherosclerosis through miR-21-3p/PTEN-mediated VSMC migration and proliferation," Theranostics, vol. 9, no. 23, pp. 6901-6919, 2019.

[14] C. M. Adams, S. W. Hiebert, and C. M. Eischen, "Myc induces miRNA-mediated apoptosis in response to HDAC inhibition in hematologic malignancies," Cancer Research, vol. 76, no. 3, pp. 736-748, 2016.

[15] L. Yu, H. Shu, L. Xing et al., "Silencing long non-coding RNA NEAT1 suppresses the tumorigenesis of infantile hemangioma by competitively binding miR-33a-5p to stimulate HIF $1 \alpha /$ NF- $\kappa$ B pathway," Molecular Medicine Reports, vol. 22, no. 4, pp. 3358-3366, 2020.

[16] Q. Z. Wang, Z. L. Zhao, C. Liu, and J. W. Zheng, "Exosomederived miR-196b-5p facilitates intercellular interaction in infantile hemangioma via down-regulating CDKN1B," Annals of Translational Medicine, vol. 9, no. 5, p. 394, 2021.

[17] M. I. Love, W. Huber, and S. Anders, "Moderated estimation of fold change and dispersion for RNA-seq data with DESeq2," Genome Biology, vol. 15, no. 12, p. 550, 2014.

[18] K. J. Livak and T. D. Schmittgen, "Analysis of Relative Gene Expression Data Using Real-Time Quantitative PCR and the $2^{-\Delta \Delta C}$ Tethod," Methods, vol. 25, no. 4, pp. 402-408, 2001.

[19] Y. Hu, S. S. Rao, Z. X. Wang et al., "Exosomes from human umbilical cord blood accelerate cutaneous wound healing through miR-21-3p-mediated promotion of angiogenesis and fibroblast function," Theranostics, vol. 8, no. 1, pp. 169-184, 2018.

[20] Y. Wang, H. Xu, L. Si et al., "MiR-206 inhibits proliferation and migration of prostate cancer cells by targeting CXCL11," Prostate, vol. 78, no. 7, pp. 479-490, 2018.

[21] Z. Xu, C. Zhu, C. Chen et al., "CCL19 suppresses angiogenesis through promoting miR-206 and inhibiting met/ERK/elk1/HIF-1 $\alpha$ /VEGF-A pathway in colorectal cancer," Cell Death \& Disease, vol. 9, no. 10, p. 974, 2018.

[22] T. Kunej, I. Godnic, J. Ferdin, S. Horvat, P. Dovc, and G. A. Calin, "Epigenetic regulation of microRNAs in cancer: an integrated review of literature," Mutation Research, vol. 717, no. 12, pp. 77-84, 2011.

[23] F. Zhao, X. Yang, G. Xu, J. Bi, R. Lv, and R. Huo, "Propranolol suppresses HUVEC viability, migration, VEGF expression, and promotes apoptosis by downregulation of miR-4295," Journal of Cellular Biochemistry, vol. 120, no. 4, pp. 66146623, 2019.

[24] P. Mansuy, N. Mougenot, J. F. Ramirez-Gil et al., "Effects of prolonged propranolol treatment on left ventricular remodeling and oxidative stress after myocardial infarction in rats," Journal of Cardiovascular Pharmacology, vol. 35, no. 5, pp. 806-813, 2000.

[25] M. Liao and L. Peng, "MiR-206 may suppress non-small lung cancer metastasis by targeting CORO1C," Cellular \& Molecular Biology Letters, vol. 25, no. 1, p. 22, 2020.
[26] C. Liu, J. Li, W. Wang, X. Zhong, F. Xu, and J. Lu, “miR-206 inhibits liver cancer stem cell expansion by regulating EGFR expression," Cell Cycle, vol. 19, no. 10, pp. 1077-1088, 2020.

[27] G. M. Strub, A. L. Kirsh, M. E. Whipple et al., "Endothelial and circulating C19MC microRNAs are biomarkers of infantile hemangioma," JCI Insight, vol. 1, no. 14, article e88856, 2016.

[28] D. Li, P. Li, Z. Guo, H. Wang, and W. Pan, "Downregulation of miR-382 by propranolol inhibits the progression of infantile hemangioma via the PTEN-mediated AKT/mTOR pathway," International Journal of Molecular Medicine, vol. 39, no. 3, pp. 757-763, 2017.

[29] E. F. Mong, K. M. Akat, J. Canfield et al., "Modulation of LIN28B/Let-7 signaling by propranolol contributes to infantile hemangioma involution," Arteriosclerosis, Thrombosis, and Vascular Biology, vol. 38, no. 6, pp. 1321-1332, 2018.

[30] H. K. Kim, Y. S. Lee, U. Sivaprasad, A. Malhotra, and A. Dutta, "Muscle-specific microRNA miR-206 promotes muscle differentiation," The Journal of Cell Biology, vol. 174, no. 5, pp. 677687, 2006.

[31] C. Y. Lin, H. C. Lee, C. Y. Fu et al., "MiR-1 and miR-206 target different genes to have opposing roles during angiogenesis in zebrafish embryos," Nature Communications, vol. 4, no. 1, p. 2829, 2013.

[32] N. Kondo, T. Toyama, H. Sugiura, Y. Fujii, and H. Yamashita, "miR-206 expression is down-regulated in estrogen receptor alpha-positive human breast cancer," Cancer Research, vol. 68, no. 13, pp. 5004-5008, 2008.

[33] H. Mataki, N. Seki, T. Chiyomaru et al., "Tumor-suppressive microRNA-206 as a dual inhibitor of MET and EGFR oncogenic signaling in lung squamous cell carcinoma," International Journal of Oncology, vol. 46, no. 3, pp. 1039-1050, 2015.

[34] R. Samaeekia, V. Adorno-Cruz, J. Bockhorn et al., "miR-206 inhibits stemness and metastasis of breast cancer by targeting MKL1/IL11 pathway," Clinical Cancer Research, vol. 23, no. 4, pp. 1091-1103, 2017.

[35] G. D'Angelo, H. Lee, and R. I. Weiner, "cAMP-dependent protein kinase inhibits the mitogenic action of vascular endothelial growth factor and fibroblast growth factor in capillary endothelial cells by blocking Raf activation," Journal of Cellular Biochemistry, vol. 67, no. 3, pp. 353-366, 1997.

[36] D. J. Hicklin and L. M. Ellis, "Role of the vascular endothelial growth factor pathway in tumor growth and angiogenesis," Journal of Clinical Oncology, vol. 23, no. 5, pp. 1011-1027, 2005.

[37] H. Shen, X. Yu, F. Yang et al., "Reprogramming of normal fibroblasts into cancer-associated fibroblasts by miRNAsmediated CCL2/VEGFA signaling," PLoS Genetics, vol. 12, no. 8, article e1006244, 2016.

[38] X. Gao, G. W. Leone, and H. Wang, "Cyclin D-CDK4/6 functions in cancer," Advances in Cancer Research, vol. 148, pp. 147-169, 2020.

[39] A. Hossian, M. S. Sajib, P. E. Tullar, C. M. Mikelis, and G. Mattheolabakis, "Multipronged activity of combinatorial miR-143 and miR-506 inhibits lung cancer cell cycle progression and angiogenesis in vitro," Scientific Reports, vol. 8, no. 1, p. 10495, 2018.

[40] Z. Zhang, J. Li, Y. Huang et al., "Upregulated miR-1258 regulates cell cycle and inhibits cell proliferation by directly targeting E2F8 in CRC," Cell Proliferation, vol. 51, no. 6, article e12505, 2018. 
[41] S. Morales, M. Monzo, and A. Navarro, "Epigenetic regulation mechanisms of microRNA expression," Biomolecular Concepts, vol. 8, no. 5-6, pp. 203-212, 2017.

[42] G. Heller, C. Altenberger, I. Steiner et al., "DNA methylation of microRNA-coding genes in non-small-cell lung cancer patients," The Journal of Pathology, vol. 245, no. 4, pp. 387398, 2018.

[43] F. Wang, Y. Ma, H. Wang, and H. Qin, "Reciprocal regulation between microRNAs and epigenetic machinery in colorectal cancer," Oncology Letters, vol. 13, no. 3, pp. 1048-1057, 2017.

[44] Z. Li, H. Lei, M. Luo et al., "DNA methylation downregulated mir-10b acts as a tumor suppressor in gastric cancer," Gastric Cancer, vol. 18, no. 1, pp. 43-54, 2015.

[45] X. Zeng, X. Qu, C. Zhao et al., "FEN1 mediates miR-200a methylation and promotes breast cancer cell growthviaMET and EGFR signaling," The FASEB Journal, vol. 33, no. 10, pp. 10717-10730, 2019. 